\title{
Describing health service areas: a methodological note on a summary indicator
}

\author{
Bonnie J. Kay \\ Department of Health Planning and Administration, University of Michigan, Ann Arbor, MI, U.S.A.
}

(Accepted for publication 27 May 1985)

Summary

This paper describes the development of an indicator of use in the analysis of regional travel patterns for health care services. The indicator is derived using two different models which describe the relationship between utilization and travel distance. Results from both models are compared in terms of their validity, sensitivity and usefulness in describing health service areas and to population-based policy analysis concerned with access-to-care for rural populations.

travel distance; utilization; access indicator; rural health

The spatial dimension of the analysis of access to health care services has received persistent attention by health policy planners over the years. An extensive literature exists which describes and analyzes the effects of physical distance on health service utilization [1-5]. The inverse relationship between travel distance and use for rural populations is well documented and a number of mathematical models have been adapted from the geographical literature on spatial behavior of consumers for explanatory purposes [6-9]. Because of the urban concentration of many health resources, the disparity in geographic access between urban and rural populations has been an historically important issue. Describing the outcomes of strategies which attempt to address this disparity is a task central to the analysis of access-to-care policies.

This paper describes an indicator of use in depicting the distribution of distances traveled by rural clients to urban centers for health care. If utilization (or some modification of it) is plotted against travel distance, the straight line representing the

Address for correspondence: Dr. B.J. Kay, Department of Health Planning and Administration, University of Michigan, Ann Arbor, Michigan 48109-2029, U.S.A. 
best fit of all data points is an approximation of the distribution of distances traveled to receive care. The line, if rotated around a point at the urban center, describes the area served by that center. The slope of the line becomes a summary indicator of the travel distance distribution.

If the utilization measure is represented by the fraction of clients traveling a specific distance $X_{i}$ to an urban center of all clients receiving care, changes in the slope directly reflect changes in this fraction. The fraction is a modification of the commitment index as defined by Griffith [10]*. Two models, one linear, one non-linear, are used to describe the relationship between utilization and travel distance and to calculate the line slope. The results are compared with a categorical approach to analyzing travel distance patterns and service area changes.

\section{Problem application}

The system of ambulatory clinics which delivers the vast majority of elective abortion services in the United States is almost entirely urban based. While many new providers have established clinics over the past eleven years since abortion laws were liberalized in the U.S., thcy have donc so ovcrwhclmingly in sclected mctropolitan areas. For rural women, getting an abortion means traveling long distances. Travel patterns frequently resemble those of specialized hospital-based services. From a public health perspective, reducing the access disparity between urban and rural women has been a continuing issue of concern although attempts to shorten travel distance for rural women by encouraging rural hospitals to offer elective abortions have met with little success [11]. In the meantime, a competitive situation has evolved among many urban-based free-standing abortion clinics which, after need has been met within the immediate area, must attract clients from further distances in order to maintain revenues in a highly inflationary medical system**. Some evidence suggests that clinics target specific populations, often geographically defined, for direct advertising and for establishing individual physician referral networks [12]. The extent of this outreach has some potential for increasing access for rural women due to the fact that travel distance barriers are compounded by a lack of information about available providers [5]. Describing changes in the geographical area served by clinics in urban centers is central to an analysis of the degree to which urban-based providers have been able to meet the needs of rural women.

\section{Method}

A patient origin study was conducted for clients traveling to three clinics in a

* The commitment index has been used to define the service area of a hospital and looks at how much a facility depends on a specific geographic area (for example, a census tract or county) for patients. It is calculated by dividing the number of discharges from hospital $X$ to area $Y$ by the total number of discharges from hospital $\mathrm{X}$.

** Prices charged for elective abortions have actually decreased in real dollar terms since 1973 [11]. 
southeastern city (city A) to obtain elective abortions during the period 1975-1979. The three clinics provided nearly $90 \%$ of all first trimester procedures in the city during this time interval. City A, population approximately 190000 , is located in a predominantly rural area.

County-of-residence data for each client were abstracted from health department records. Because specific addresses were not available for reasons of protecting client identity, it was necessary to approximate the actual distances traveled by clients. An average distance for each county to the center of city A was calculated by summing the weighted distances of each population center within the county. Weights represented the fraction of residents residing in a center $j$ of all residents of incorporated towns and unincorporated towns in the county for which population counts were available. This procedure presumes that residences of abortion clients are distributed throughout a county in the same manner as those of the general population. County-to-city travel distance is expressed as

$$
\begin{aligned}
& \text { distance }_{i}=w_{l i} x_{l i}+w_{2 i} x_{2 i}+\ldots \text { for each county } i \\
& w_{j i}=\frac{p_{j i}}{\sum_{j} p_{j i}} \text { for each county } i
\end{aligned}
$$

where $p_{j i}=$ population in center $j$ for each county $i$; and $x_{j i}=$ shortest highway distance from center $j$ in county $i$ to city A.

The areas of counties involved in this analysis are relatively small and the majority of the population is clustered in two or three towns. The county seat is often located close to the geographical center of each county. Using it to approximate a travel origin point to the city could represent a simpler method for approximating travel distance. Comparative results of the weighted distance measure and the county seat approximation are presented in Table 2 in a later section of this paper.

A modified commitment index for city $A$ was calculated by dividing the number of clients traveling from each county $i$ at a distance $X_{i}$ from the city by the total number of clients attending the three clinics. This fraction is the proportion of women using abortion services in city $\mathrm{A}$ who have traveled $X_{i}$ miles to receive those services.

\section{Linear model}

By assuming a linear relationship between distance and utilization, values of the commitment index for various travel distances can be estimated by the following equation:

$$
\mathrm{CI}_{i}=a+b X_{i}
$$

where $\mathrm{CI}_{i}=$ (number of clients traveling distance $X_{i} /$ total number of clients) $\times 100 ; X_{i}$ $=$ distance traveled to city A from county $i$; and $a, b=$ constants. Estimates for $a$ and $b$ are obtained by linear regression. The magnitude of $b$, the slope of the line, is a 
reflection of the size of the service area. A large negative value would signify a relatively small, urban-focussed service area while a less negative value of the slope would represent a larger area which included a larger fraction of rural clients.

\section{Non-linear (gravity) model}

The geographic literature suggests that the relationship between distance and utilization is better approximated by an exponential function, particularly as applied to rural areas. Based on an application of Reilly's law of retail gravitation, this relationship can be expressed as follows:

$$
\mathrm{CI}_{i}=a_{\mathrm{g}} X_{i}^{b_{\mathrm{g}}}
$$

where $\mathrm{CI}_{i}$ and $X_{i}$ are defined as before and $a_{\mathrm{g}}$ and $b_{\mathrm{g}}$ are constants. By taking the logarithm of both sides of this equation, the constant $b_{\mathrm{g}}$ can be interpreted in the same manner as for the linear model. That is:

$$
\log \mathrm{CI}_{i}=\log a_{\mathrm{g}}+b_{\mathrm{g}} \log X_{i}
$$

Estimates for $b_{\mathrm{g}}$ are obtained from a linear regression of $\log X_{i}$ on $\log \mathrm{CI}_{i}$.

\section{Validity}

Table 1 shows the results of calculations of the slope indicators $b$ and $b_{\mathrm{g}}$ for the two models for each year of the 1975-1979 period. These are contrasted with a categorical indicator defined as the percentage of all clients traveling 20 miles or less to city A. Two points of difference are apparent. Using the 20 mile category as a proxy for urban clients, a slight increase $(+1.2 \%)$ in the fraction of urban clients served by city $\mathrm{A}$ occurred from 1975 to 1976 . This fraction subsequently decreased uniformly throughout the remainder of the analysis period. Values of the slope indicator derived from the linear model became less negative consistently during the entire period. Values derived from the gravity model followed this same pattern year-to-year except for the last interval, 1978-1979, when the slope became more negative*. Thus, using the slope indicators, an increasing annual fraction of rural clients is depicted. The linear model shows a consistent increase while the gravity model exhibits an increase until 1978-1979 when it depicts a shift to a larger share of urban clients. The slope indicator derived from the linear model is in better (although not complete) agreement with the

* The 1975-76 difference reflects the fact that one of the three clinics, with a predominantly urban caseload, was in operation for only part of 1975 . Totals for 1976 represent a full year caseload. Both slope indicator models weighed this effect less than did the categorical model.

For $1978-79$, the actual total number of cases increased from 4132 to 4806 , a $16.3 \%$ increase. More of this increase came from women traveling 21 miles or more (a 18.9\% increase) compared with those traveling 20 miles or less (a $13.1 \%$ increase). The gravity model weighs the latter cases more heavily. This is reflected in the value of the slope $b_{\mathrm{g}}$ which became more negative during the 1978-79 interval. 


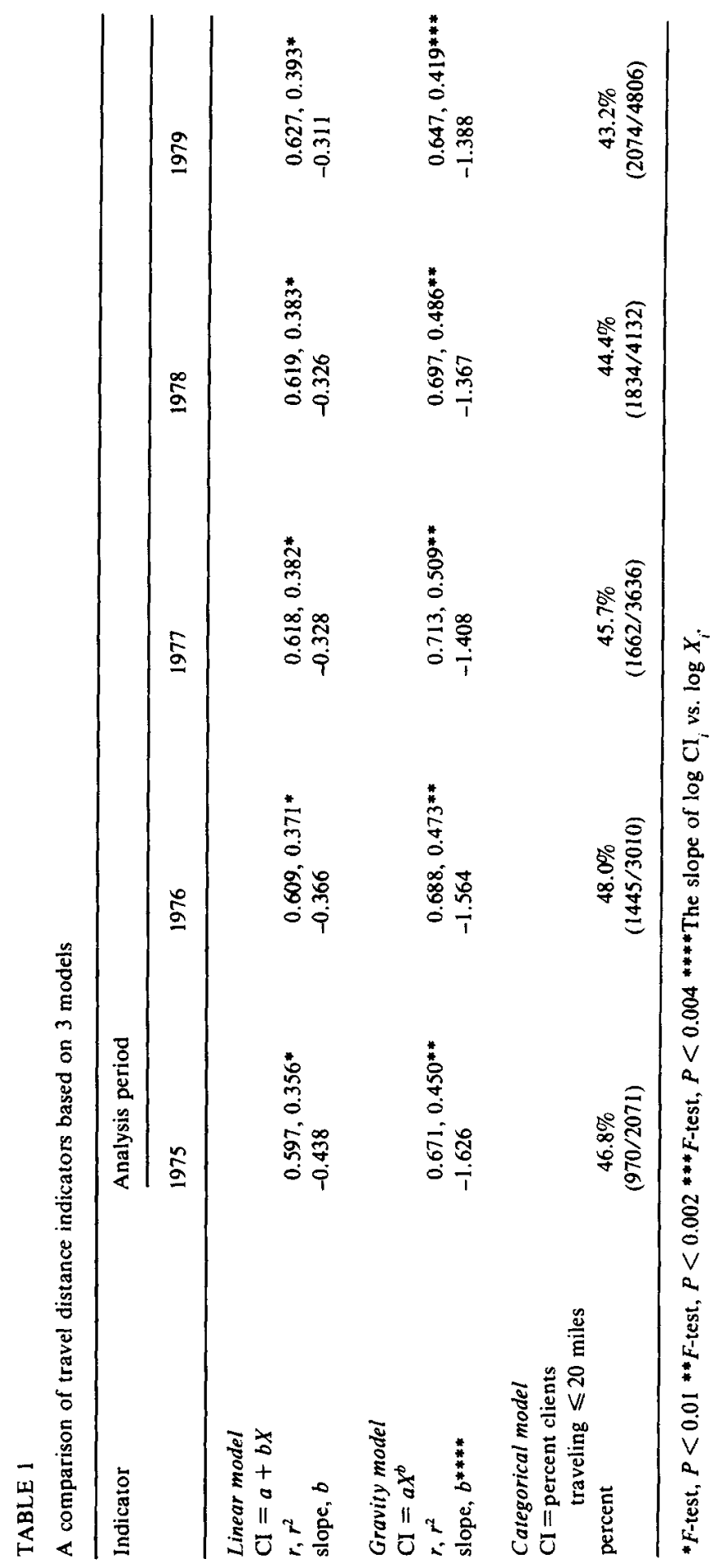


categorical indicator than is that derived using the gravity model. It should also be noted that the trend depicted by the percentage of clients traveling 20 miles or less provides no information about the distribution of clients beyond the 20 mile category. The slope indicator avoids the issue of how to define distance categories by representing the entire distribution of travel distances.

How strong is the relationship between distance and utilization? Average values of the five year period for the correlation coefficient $(r)$ and coefficient of variation $\left(r^{2}\right)$ for the linear model are 0.614 and 0.377 respectively. Corresponding values for the gravity model are 0.683 and 0.464 . Each is significantly different from zero. A higher percentage of variance in the commitment index is explained by the gravity model (averages of $46.7 \%$ compared with $37.7 \%$ ). If the county seat is used as the travel origin point to approximate travel distance, similar results are obtained. Both the weighted distance method and the county seat travel origin approximation are in agreement in terms of trends from year to year as depicted in Table 2. The strength of association between distance and utilization is significantly greater than zero $(P<0.01)$ for both linear and gravity models. However, while travel distance accounts for a relatively large share of the variation in the commitment index value as a single variable, at least half the variation must be accounted for by other variables. With this limitation in mind, the sensitivity of the slope indicator to shifts in the fraction of urban and rural clients is examined next.

\section{Sensitivity}

The sensitivity of categorical, linear and gravity model-based indicators was compared by recalculating their values after shifting $20 \%$ of clients traveling from within city $A$ to distances greater than 50 miles. Table 3 presents these data. While the percentage variance in the commitment index explained by both linear and gravity models decreases with this shift, $r$ values remain significantly different than zero $(P<$ 0.02). Values of the slope, $b$, become less negative, agreeing with the direction of the change and reflecting a larger share of rural clients. A $20 \%$ shift of clients to travel distances greater than 50 miles results in a $26.8 \%$ decrease in the slope for the linear model and a $21.3 \%$ decrease in the slope for the gravity model. Thus, the slopes resulting from either of these models are slightly more sensitive to population shifts than the categorical indicator.

\section{Feasibility, clarity and applicability}

Data requirements for calculating the slope indicator $b$ are the same as those required for patient origin studies generally. The availability of mini-computer systems and the ease of use of statistical software programs which include least-squares regression make the additional labor required in calculating estimates of $b$ minor.

The problem posed in this paper considered the dependence of three providers collectively on the surrounding community for clients. The slope indicator could be 


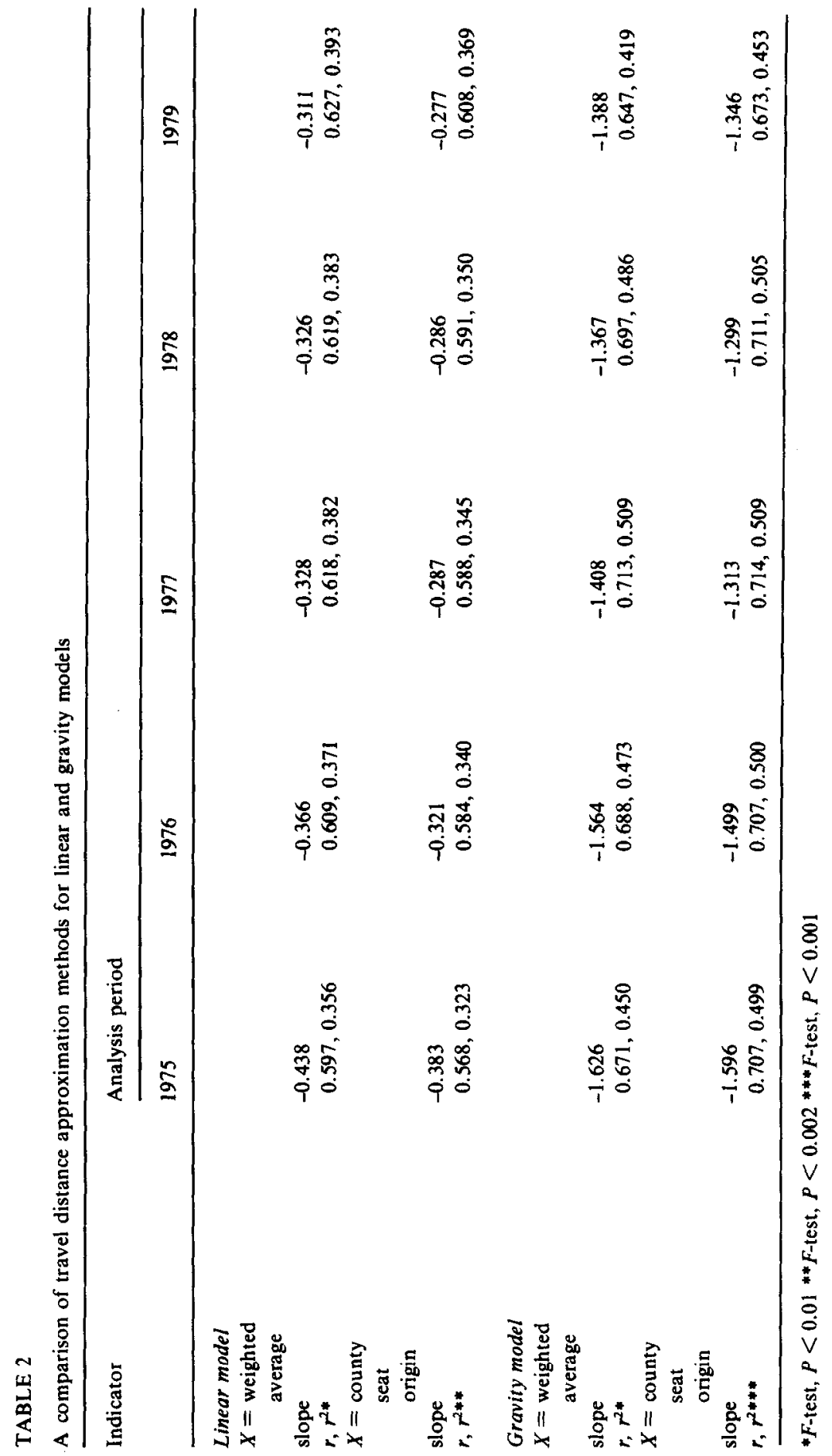


TABLE 3

Sensitivity of indicators to a $20 \%$ urban to rural shift in travel distances (1977)

\begin{tabular}{llll}
\hline Indicator & Before shift & & After shift \\
\hline $\begin{array}{l}\text { Linear model } \\
b\end{array}$ & -0.328 & 26.8 & -0.240 \\
$\%$ change & & & \\
$\begin{array}{l}\text { Gravity model } \\
b\end{array}$ & -1.408 & 21.3 & -1.108 \\
$\%$ change & & & \\
$\begin{array}{l}\text { Categorical model } \\
\text { No. traveling } \leqslant 20 \text { miles/total clients) } \times 100\end{array}$ & 45.7 & & 36.5 \\
$\%$ change & & 20.0 & \\
\hline
\end{tabular}

useful in comparing service area characteristics between providers as well. For example, a comparison of $b$ values calculated using the linear model for each of the three clinics reveals the extent to which each provider depends on urban (or rural) clients relative to other providers. For $1977, b$ values for clinics 1,2 , and 3 are $-0.200,-0.388$, and -0.272 , respectively. Clinic 1 is relatively more dependent on a rural clientele, clinic 2 on an urban clientele and clinic 3 represents an intermediate case. Comparing changes in $b$ over time allows the analyst to track changes in the proportion of rural and urban clients served by each clinic relative to the others.

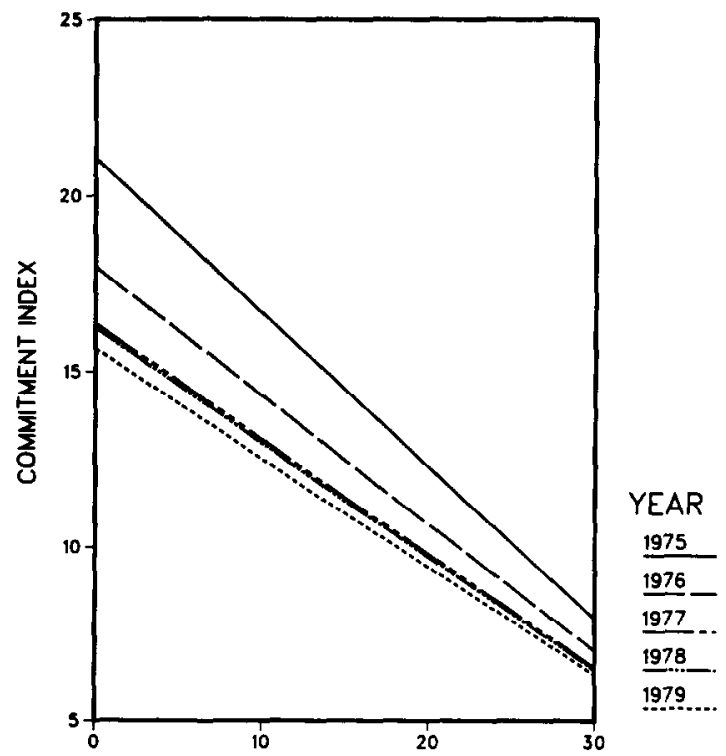

Fig. 1 TRAVEL DISTANCE-MILES

Fig. 1. Travel patterns: slope indicator. $b_{75}=-0.438 ; b_{76}=-0.366 ; b_{77}=-0.328 ; b_{78}=-0.326 ; b_{79}=-0.311$. 
Figure 1 illustrates graphically the relationship between the slope indicator $b$ and travel distance distributions derived using the linear model. While the gravity model does a better job of approximating the relationship between travel distance and utilization, the interpretation of $b$ is less direct. It is for this reason that an estimate of $b$ derived from regressing travel distance directly on the commitment index is more useful as a summary indicator. Data depicted in Figure 1 show travel distributions for the years 1975 through 1979 as summarized by $b$ values for each year. Such types of analysis comprise an important share of the regional health analyst's work in evaluating policy proposals which necessitate population-based analysis.

\section{References}

I Shannon, G., Bashur, R. and Metzner, C., The concept of distance as a factor in accessibility and utilization of health care, Medical Care Review, 26 (1969) 143-161.

2 Shannon, G. and Dever, G., Health Care Delivery: Spatial Perspectives, McGraw Hill, New York, 1974.

3 Weiss, J., Greenlick, M. and Jones, J., Determinants of medical care utilization: The impact of spatial factors, Inquiry, 8 (1971) 50-57.

4 Kleinman, I. and Makuc, D., Travel for ambulatory medical ca re, Medical Care, 21 (1983) $543-557$.

5 Shelton, J., Brann, E. and Schulz, K., Abortion utilization: does travel distance matter? Family Planning Perspectives, 8 (1976) 260-263.

6 Shannon, G. and Dever, G., Health Care Delivery, Chapter V: Impact of Geographic Factors on Health Care, McGraw-Hill, New York, 1974, pp. 89-109.

7 Stock, R., Distance and the utilization of health facilities in rural Nigeria, Social Science and Medicine, 17 (1983) 563-570.

8 Meade, J., A mathematical model for deriving hospital service areas, International Journal of Health Services Research, 4 (1974) 353-364.

9 Shonick, W., In Elements of Planning for Area-wide Personal Health Services, chapt. 6, Location Optimization - Distributing the Anticipated Utilization Among Facilities, C.V. Mosby Co., St. Louis, 1976, pp. 61-78.

10 Griffith, J., Quantitative Techniques for Hospital Planning and Control, D.C. Heath, Lexington, MA, 1972, pp. 75-78.

11 Henshaw, S., Forrest, J. and Blaine, E., Abortion services in the United States, 1981 and 1982, Family Planning Perspectives, 16 (1984) 119-127.

12 Kay, B., Whitted, N. and Hardin, S., An economic interpretation of the distribution and organization of abortion services, Inquiry, 18 (1981) 322-331. 